\title{
Clinical Evidence for a Mandibular to Maxillary Transformation in Auriculocondylar Syndrome
}

\author{
Christopher T. Gordon, ${ }^{1 *}$ Christopher M. Cunniff, ${ }^{2}$ Glenn E. Green, ${ }^{3}$ Roseli Maria Zechi-Ceide, ${ }^{4}$ \\ Jason M. Johnson, ${ }^{5}$ Alex Henderson, ${ }^{6}$ Florence Petit, ${ }^{7}$ Nancy Mizue Kokitsu-Nakata, ${ }^{4}$ \\ Maria Leine Guion-Almeida, ${ }^{4}$ Arnold Munnich, ${ }^{1,8}$ Michael L. Cunningham, ${ }^{9,10}$ Stanislas Lyonnet, ${ }^{1,8}$ \\ and Jeanne Amiel ${ }^{1,8 * *}$ \\ ${ }^{1}$ INSERM U1163, Hôpital Necker-Enfants Malades and Université Paris Descartes-Sorbonne Paris Cité, Institut Imagine, Paris, France \\ ${ }^{2}$ Department of Pediatrics, University of Arizona, Tucson, Arizona \\ ${ }^{3}$ Department of Otolaryngology-Head \& Neck Surgery, University of Michigan, Ann Arbor, Michigan \\ ${ }^{4}$ Department of Clinical Genetics, Hospital for Rehabilitation of Craniofacial Anomalies, University of São Paulo (HRCA-USP), Bauru, Brazil \\ ${ }^{5}$ Division of Neuroradiology, Department of Radiology, University of California San Francisco, San Francisco, California \\ ${ }^{6}$ Northern Genetics Service, Newcastle upon Tyne Hospitals NHS Foundation Trust, Newcastle Upon Tyne, UK \\ 'Service de Génétique Clinique, Hôpital Jeanne de Flandre, CHRU Lille, France \\ ${ }^{8}$ Département de Génétique, Hôpital Necker-Enfants Malades, AP-HP, Paris, France \\ ${ }^{9}$ Center for Developmental Biology and Regenerative Medicine, Seattle Children's Research Institute, Seattle, Washington \\ ${ }^{10}$ Division of Craniofacial Medicine, Department of Pediatrics, University of Washington \& Seattle Children's Hospital Craniofacial Center, \\ Seattle, Washington
}

Manuscript Received: 8 October 2013; Manuscript Accepted: 25 January 2014

\section{TO THE EDITOR:}

Auriculocondylar syndrome (ACS, OMIM 602483 and 614669) is a rare craniofacial disorder affecting derivatives of the first and second branchial arches (BAs), and is characterized by mandibular hypoplasia, condylar abnormalities, microstomia, full cheeks, and a distinctive anomaly of the pinnae involving variable constriction between the lobe and helix, known as a question mark ear (QME). The majority of ACS patients harbor heterozygous missense mutations in phospholipase C, beta 4 (PLCB4) and guanine nucleotide binding protein ( $\mathrm{G}$ protein), alpha inhibiting activity polypeptide 3 (GNAI3) [Rieder et al., 2012; Gordon et al., 2013]. In the early embryo the first BA is divided into maxillary and mandibular prominences, which give rise to the skeletal elements and soft tissues of the upper and lower jaws, respectively. PLCB4 and GNAI3 are predicted to act within the endothelin 1 (EDN1)-

\section{How to Cite this Article:}

Gordon CT, Cunniff CM, Green GE, Zechi-

Ceide RM, Johnson JM, Henderson A, Petit

F, Kokitsu-Nakata NM, Guion-Almeida

ML, Munnich A, Cunningham ML,

Lyonnet S, Amiel J. 2014. Clinical evidence

for a mandibular to maxillary

transformation in Auriculocondylar

syndrome.

Am J Med Genet Part A 164A:1850-1853.

The authors have no conflict of interest to declare.

Grant sponsor: Université Sorbonne Paris-Cité Pôle de recherche et d'enseignement supérieur; Grant number: SPC/JFG/2013-031; Grant sponsor: ANR project EvoDevoMut 2010; Grant sponsor: E-Rare CRANIRARE.

*Correspondence to:

Christopher T. Gordon, INSERM U1163, Institut Imagine, 24 Boulevard du Montparnasse, 75015 Paris, France.

E-mail: chris.gordon@inserm.fr

** Correspondence to:

Jeanne Amiel, INSERM U1163, Institut Imagine, 24 Boulevard du Montparnasse, 75015 Paris, France.

E-mail: jeanne.amiel@inserm.fr

Article first published online in Wiley Online Library

(wileyonlinelibrary.com): 26 March 2014

DOI 10.1002/ajmg.a.36505 
endothelin receptor type A (EDNRA) signaling pathway, which plays a crucial role in specifying the identity of mandibular-derived structures in animal models. The severe defects of the mandibular skeleton in mice deleted for Edn1, Ednra or Dlx5 and Dlx6 (target genes of the Edn1-Ednra pathway) represent a loss of mandibular identity and a gain of maxillary identity, that is, a homeotic transformation [Beverdam et al., 2002; Depew et al., 2002; Ozeki et al., 2004; Ruest et al., 2004]. The implication of PLCB4 and GNAI3 in the EDN1-EDNRA pathway and the morphology of the mandibular skeleton in ACS patients led to the hypothesis that ACS also represents a mandibular to maxillary transformation [Rieder et al., 2012]. Expressivity within ACS families is highly variable, with some mutation-positive individuals presenting a relatively normal mandible, or ears, or both. Here we describe an abnormal distribution of facial hair in adult ACS males that we consider as a further manifestation of the mandibular to maxillary transformation thought to underlie ACS. This finding highlights how the endothelin pathway influences the development of both the mandibular skeleton and the overlying mandibular ectoderm.

Figure 1 depicts four individuals known or suspected to harbor a mutation in PLCB4 or GNAI3. The individual in panel A is S006-P in Rieder et al. [2012], who displays mild mandibular hypoplasia while his two children have classic ACS, all three with the PLCB4 substitution p.Asn329Ser. Panel B depicts the mildly affected father of case 5 in Gordon et al. [2013], with the PLCB4 substitution p. Arg621Leu. Panel C shows a mildly affected individual from family M001 in Rieder et al. [2012], with the PLCB4 substitution p. Arg621His. The ACS individual in panel D is a member of a previously published family [Guion-Almeida et al., 2002], for whom the mutation has not been reported. However, linkage analysis of ACS-affected individuals in this family showed that the phenotype segregates with an interval on chromosome 1 harboring GNAI3 [Masotti et al., 2008]. All four individuals above displayed an absence of facial hair growth in regions along and/or beneath the jaw-line, extending from the ear to, but not including, the chin (Fig. 1). We suspect a similar phenotype in the individual shown in panels Civ-vi of Figure 2 in Gordon et al. [2013] who harbors the GNAI3 substitution p.Ser47Arg; note the wide band of stubble across his cheeks, but not along or below the jaw-line, in those images (we have been unable to obtain images of more advanced facial hair growth of this individual). We also analyzed facial hair growth in two adult males (III:1 and III:4) of the PLCB4 mutation-positive family M003 in Rieder et al. [2012]; III:4 presented QMEs and a region lacking hair midway along the jaw-line, while III:1 showed neither mandibular defects, QME nor a facial hair phenotype (data not shown). Interestingly, most of the indi-
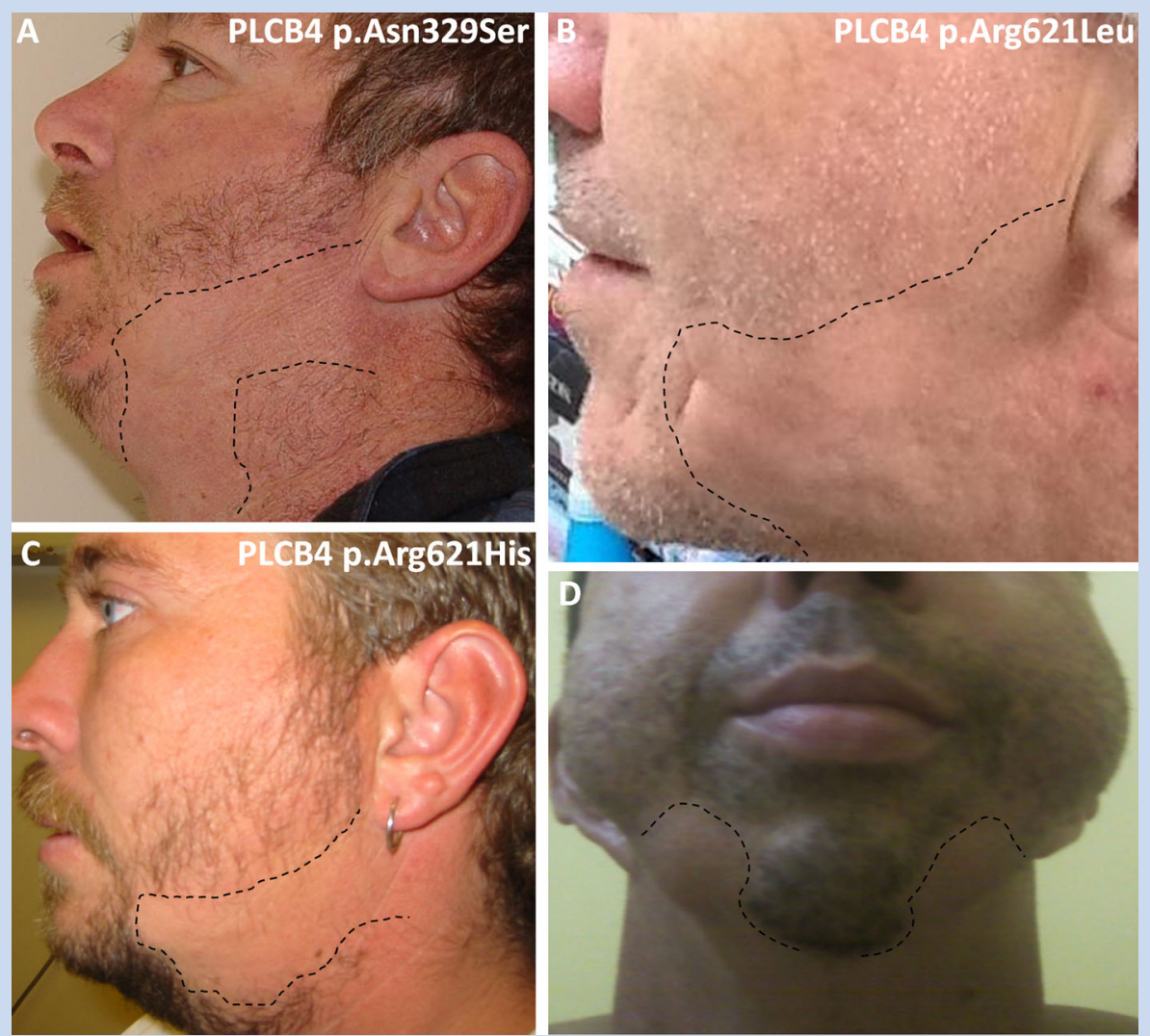

FIG. 1. Facial hair distribution in adult ACS males. The previously published PLCB4 substitution is indicated for individuals in panels A-C, while the ACS phenotype in the family of the individual in panel $D$ has been mapped to an interval harboring GNAI3 [see text for details]. Hairless zones are demarcated by dashed lines. In B, stubble is poorly visible but is restricted to regions above and to the left of the dotted line. 


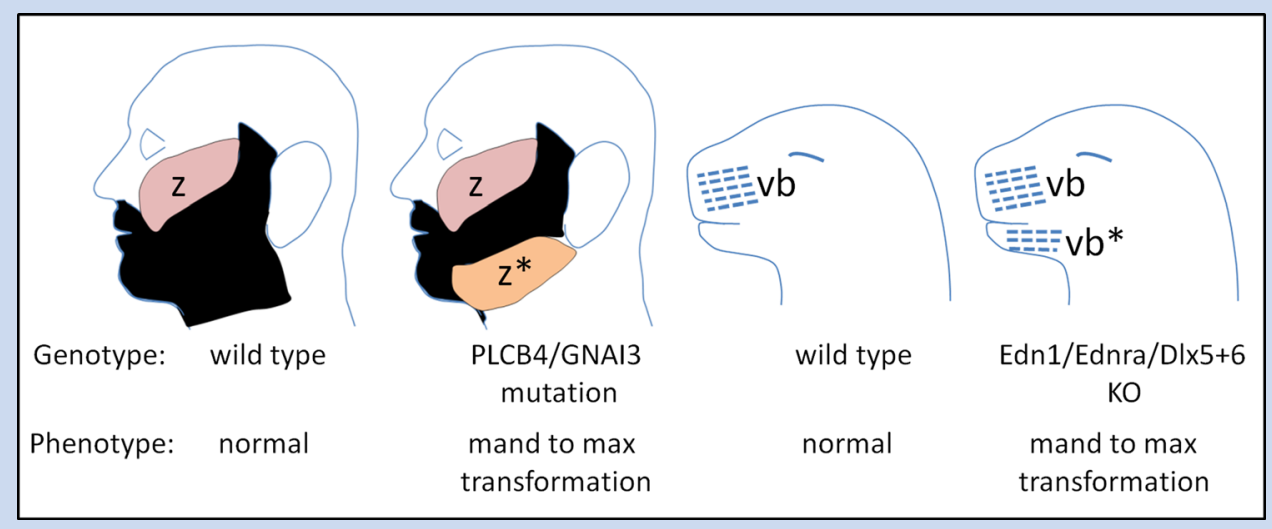

FIG. 2. Schematic comparing the proposed ectodermal manifestations of mandibular to maxillary transformations in mice and men. $z$, hairless skin covering zygomatic arch; vb, vibrissae. An asterisk indicates ectopic zygomatic arch skin on the mandible of ACS males, or ectopic vibrissae on the mandible of mice null for components of the endothelin signaling pathway.

viduals presenting a facial hair phenotype had relatively normal mandibular structure and ears, suggesting that the facial hair abnormality is a relatively highly penetrant component of the ACS phenotype.

In addition to a mirror image version of upper jaw skeletal elements in the mandible of mice deleted for $E d n 1, E d n r a$ or Dlx5 and $D l x 6$, the lower jaw ectoderm also appears to undergo a transformation, with the appearance of ectopic mandibular vibrissae, sensory hairs normally restricted to the skin of the upper jaw (Fig. 2) [Beverdam et al., 2002; Depew et al., 2002; Ozeki et al., 2004; Ruest et al., 2004]. Ectopic mandibular vibrissae were also observed in mice deleted for Hand2, a Dlx5/Dlx6 target gene [Barron et al., 2011]. Therefore, mandibular identity as specified by the Edn1-Ednra pathway in mice involves the inhibition of development of these ectodermal appendages. In human males, mandibular identity involves the presence of hair, while most of the skin covering the zygomatic arch is hairless. Therefore we hypothesize that the disruption of the EDN1-EDNRA pathway in ACS results in a suppression of mandibular ectodermal identity, leading to a mirror image version of the upper jaw facial hair program, that is, hairlessness, on the mandible. This implies that the maxillary program is the default state for both the maxillary and mandibular prominences, and that the EDN1-EDNRA pathway is required in the mandible to break this symmetry. Interestingly, all individuals with reduced hair growth on regions of the mandible nonetheless maintained normal growth on the chin, that is, the most distal portion of the mandible. Studies in mice have suggested that proximal regions of the mandible are more sensitive to defects in endothelin signaling than the distal mandible [Tavares et al., 2012]. Indeed, knock-in of the Ednrb cDNA at the Ednra locus in Ednra null mice rescues only the distal-most aspects of the mandibular skeleton, while in mice with a deletion of Gnaq and Gna11 (encoding G proteins thought to function downstream of Ednra in mice), a similar distal domain is the least affected part of the mandible [Sato et al., 2008]. As is likely in these mouse models, we speculate that the maintenance of facial hair on skin covering the chin in ACS patients reflects a reduced sensitivity of this region to loss of Ednra signaling.

In conclusion, our comparison of facial hair phenotypes in ACS patients and mice with deletions of components of the Edn1-Ednra pathway strengthens the proposed involvement of PLCB4 and GNAI3 in this pathway, and supports the existence of a lower to upper jaw identity switch as the basis of ACS. The observations presented here will likely assist in the evaluation of families with suspected ACS.

\section{ACKNOWLEDGMENTS}

This work was supported by funding from a Universite Sorbonne Paris-Cité Pôle de recherche et d'enseignement supérieur grant, project number SPC/JFG/2013-031 (JA), from the ANR for the project EvoDevoMut 2010 (SL) and from an E-Rare CRANIRARE grant (JA and SL).

\section{REFERENCES}

Barron F, Woods C, Kuhn K, Bishop J, Howard MJ, Clouthier DE. 2011. Downregulation of Dlx 5 and Dlx6 expression by Hand 2 is essential for initiation of tongue morphogenesis. Development 138:2249-2259.

Beverdam A, Merlo GR, Paleari L, Mantero S, Genova F, Barbieri O, Janvier P, Levi G. 2002. Jaw transformation with gain of symmetry after Dlx5/ Dlx6 inactivation: Mirror of the past? Genesis 34:221-227.

Depew MJ, Lufkin T, Rubenstein JL. 2002. Specification of jaw subdivisions by Dlx genes. Science 298:381-385.

Gordon CT, Vuillot A, Marlin S, Gerkes E, Henderson A, Alkindy A, Holder-Espinasse M, Park SS, Omarjee A, Sanchis-Borja M, Bdira EB, Oufadem M, Sikkema-Raddatz B, Stewart A, Palmer R, McGowan R, Petit F, Delobel B, Speicher MR, Aurora P, Kilner D, Pellerin P, Simon M, Bonnefont JP, Tobias ES, Garcia-Minaur S, Bitner-Glindzicz M, Lindholm P, Meijer BA, Abadie V, Denoyelle F, Vazquez MP, Rotky-Fast C, Couloigner V, Pierrot S, Manach Y, Breton S, Hendriks YM, Munnich A, Jakobsen L, Kroisel P, Lin A, Kaban LB, Basel-Vanagaite L, Wilson L, Cunningham ML, Lyonnet S, Amiel J. 2013. Heterogeneity of mutational 
mechanisms and modes of inheritance in auriculocondylar syndrome. J Med Genet 50:174-186.

Guion-Almeida ML, Zechi-Ceide RM, Vendramini S, Kokitsu-Nakata NM. 2002. Auriculo-condylar syndrome: Additional patients. Am J Med Genet 112:209-214.

Masotti C, Oliveira KG, Poerner F, Splendore A, Souza J, Freitas Rda S, Zechi-Ceide R, Guion-Almeida ML, Passos-Bueno MR. 2008. Auriculocondylar syndrome: Mapping of a first locus and evidence for genetic heterogeneity. Eur J Hum Genet 16:145-152.

Ozeki H, Kurihara Y, Tonami K, Watatani S, Kurihara H. 2004. Endothelin1 regulates the dorsoventral branchial arch patterning in mice. Mech Dev 121:387-395.

Rieder MJ, Green GE, Park SS, Stamper BD, Gordon CT, Johnson JM, Cunniff CM, Smith JD, Emery SB, Lyonnet S, Amiel J, Holder M, Heggie AA, Bamshad MJ, Nickerson DA, Cox TC, Hing AV, Horst JA, Cunning- ham ML. 2012. A human homeotic transformation resulting from mutations in PLCB4 and GNAI3 causes auriculocondylar syndrome. Am J Hum Genet 90:907-914.

Ruest LB, Xiang X, Lim KC, Levi G, Clouthier DE. 2004. Endothelin-A receptor-dependent and -independent signaling pathways in establishing mandibular identity. Development 131:4413-4423.

Sato T, Kawamura Y, Asai R, Amano T, Uchijima Y, Dettlaff-Swiercz DA, Offermanns S, Kurihara Y, Kurihara H. 2008. Recombinase-mediated cassette exchange reveals the selective use of Gq/G11-dependent and independent endothelin $1 /$ endothelin type A receptor signaling in pharyngeal arch development. Development 135:755-765.

Tavares AL, Garcia EL, Kuhn K, Woods CM, Williams T, Clouthier DE. 2012. Ectodermal-derived Endothelin 1 is required for patterning the distal and intermediate domains of the mouse mandibular arch. Dev Biol 371:47-56. 